\title{
Analysis of distance protection's operation in cases of deep saturation of current transformers
}

\author{
Anatoly A. Osintsev ${ }^{1, *}$, Aleksandra I. Naumova ${ }^{1}$, and Elena I. Gracheva ${ }^{2}$ \\ ${ }^{1}$ Novosibirsk State Technical University, K.Marksa prospect, 20, 630073, Novosibirsk, Russia \\ ${ }^{2}$ Kazan State Power Engineering University, Krasnoselskaya st,. 51, 420066, Kazan, Russia
}

\begin{abstract}
There is usually no information about permissible error of current transformers in modes succeed by large relative short-circuit current, at which microprocessor-based protections operate stably. By this reason, it is necessary to use data, defined for analog relays. It leads to value appreciation of a project because it is often essential to reduce current transformers' error in case of a short circuit fault. Therefore, it is necessary to define the value of current transformers' error, permitted for impedance relays. Conclusions of fundamental and applied sciences (mathematical analysis, theoretical foundations of electrical engineering, theory of simulation), analytical methods of researching nonlinear circuits and digital signal processing were used. A simulation model was created for setting overall tests of the current trans-former (CT) system. It was a relay protection device that reflected all the important properties of studied objects and allowed an analysis of digital distance protection's operation at high levels of short-circuit currents. The factors influ-encing over digital distance protection's operation in case of deep saturation of CTs were revealed, and a certain algorithm for definition of the permissible CT errors was proposed. Stable operation of digital distance protection was observed in case of a fault nearby the place of current transformers' setting in all theoretically possible combinations of electrical system's power and length of a protected electric power transmission line. It is valid if electric load choice is carried with account for stable protection's operation in condition of a fault in the computational point and if voltage swell in secondary wirings is infeasible.
\end{abstract}

\section{Introduction}

To date, microprocessor-based digital protections are widely used [1, 2]. Such protec-tions have better characteristics compared with analog protection systems. The level of short-circuit currents increases due to electricity grid development and increasing of industrial capacity. It may become the reason of current transformers saturation in conditions of a fault nearby the place of current transformers' setting. It is important to provide stable operation of relay protection in such modes [3-7]. The essence of the problem is that permissible error of current transformers, valid for analog impedance relays, is not justified in relation to digital protection, but is often used due to the lack of other data. Such method leads to increasing of protection's operation stability. By this reason, calculated secondary CT-load may seem excess, but in fact, it is permissi-ble for digital protection if the calculation is carried as for analog relays. In such cases, there is no necessity to assume additional valuable measures to reduce current trans-formers' error.

\section{Mathematical model}

To solve this problem, it is necessary to consider functioning of current transformers and measuring elements of the investigated devices in extreme modes offaults, when significant CT errors may occur, distorting secondary currents and, consequently, leading to a violation of relay protection stability. In this case, not only the current error is important, but also the angular error, which can also lead to unnecessary operation or failure in operation of protection.

Mathematical model of a part of an electrical network, which is necessary to study operation of digital distance e protection in case of a fault near CT setting, as well as all further considered objects, is created in MATLAB software (fig. 1). Investigated model consists of a part of an electrical network consists of equivalent power system $\mathrm{S}$ with inductive reactance $X_{S}$ and generated voltage $E_{S}$ and an electric power transmission line $L$ with inductive reactance $X_{L}$.

\footnotetext{
* Corresponding author: osintsev@list.ru
} 


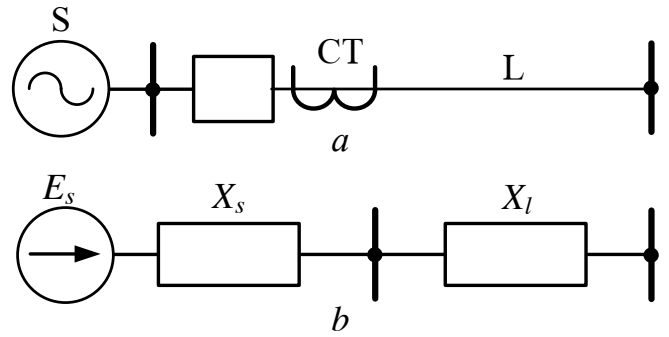

Fig. 1. Simulated part of an electrical network ( $a$ - primary electrical network, $\mathrm{b}$ - equivalent network).

To carry out a high-quality check of relay protection devices, it is necessary to obtain reliable information about shape of secondary current of a current transformer. The most difficult thing in modeling of a primary current transducer is mathematical description of the processes in its magnetic circuit, to which a large number of publications are devoted, for example [8-13]. Moreover, mathematical models of CT were developed as part of the creation of software systems for modeling electromagnetic transient processes in electric power systems in a number researches [14-16]. At the same time, mathematical description of the investigated object's operation should be as simple and clear as possible. In this regard, the following assumptions were made in the CT's model, which do not create large errors in the result:

- active and inductive resistance of the primary winding are equal to zero;

- the magnetic field inside the magnetic core is distributed regularly.

Description of the current transformer is compiled according to [17]. Saturation of the CT is taken into account by setting the magnetization curve in the form of an approximating function with selected coefficients [18].

The main parameters of current transformers (a core cross-sectional area $S_{M}$, length of mean magnetic line $l_{M}$, number of rounds of primary and secondary winding, the active resistance of the secondary winding) are determined from the passport data for a specific type of CT.

The voltage transformer model should be focused on the analysis of the processes in them in case of a fault.

Their impact on distance protection's operation is not significant. By this reason, it is enough to make scaling of primary signals' arrays, taking into account the CT ratio. The main purpose is to make an algorithm of distance protection's operation, precisely for it's measuring elements and logic section [19].

Parameters of the response characteristic are calculated automatically after entering of the line resistance value. Taking into account that nowadays manufacturers of relay protection devices provide a wide range of forms of distance protections response characteristic, we will consider only the most frequently used ones.

In the simplest case, it is a circle passing through zero of coordinate system. The diameter of the circle is equal to $85 \%$ of a protected line resistance. The inductive, active resistance of the power transmission line and the angle of maximum sensitivity are determined. A distant element with a circular characteristic passes through points $Z_{1}$ and $Z_{2}$, located at the ends of the diameter:

$$
\begin{aligned}
& Z_{1}=(0 ; 0) ; \\
& Z_{2}=\left(0,85 \cdot R_{L} ; 0,85 \cdot X_{L}\right) ; \\
& R_{L}=\frac{X_{L}}{\operatorname{tg} \phi_{L}} .
\end{aligned}
$$

The middle of the interval connecting points $Z_{1}$ and $Z_{2}$ will be the center of the circle, which is the line of the response characteristic demarcation.

We will also consider generation of a response characteristic in the form of a polygon. Currently, microprocessor-based protections manufactured by different companies, including foreign ones, are used in Russian power systems. The most frequently used terminals for the protection of electrical networks with a voltage of $110 \mathrm{kV}$ and above are manufactured by the company "Ekra" (Cheboksary) [20]. These terminals will be investigated further. Calculations of the operation settings, as well as the slope of the operation characteristic, were made according to the recommendations of the manufacturer. The measurement resistance vector $Z(n T)$ will be in the area of operation of the digital distance protection with a polygonal characteristic if the following system of inequalities correspond to the studied case:

$$
\left.\begin{array}{l}
X(n T)-\frac{X_{6}-X_{5}}{R_{6}-R_{5}} R(n T)+\frac{X_{6} R_{5}-X_{5} R_{6}}{R_{6}-R_{5}} \geq 0 ; \\
X(n T)-\frac{X_{6}-X_{5}}{R_{6}-R_{5}} R(n T)+\frac{X_{6} R_{5}-X_{5} R_{6}}{R_{6}-R_{5}} \geq 0 ; \\
X(n T)-\frac{X_{6}-X_{5}}{R_{6}-R_{5}} R(n T)+\frac{X_{6} R_{5}-X_{5} R_{6}}{R_{6}-R_{5}} \leq 0 ; \\
X(n T)-\frac{X_{6}-X_{5}}{R_{6}-R_{5}} R(n T)+\frac{X_{6} R_{5}-X_{5} R_{6}}{R_{6}-R_{5}} \leq 0 ; \\
X(n T)-\frac{X_{6}-X_{5}}{R_{6}-R_{5}} R(n T)+\frac{X_{6} R_{5}-X_{5} R_{6}}{R_{6}-R_{5}} \leq 0 ; \\
X(n T)-\frac{X_{6}-X_{5}}{R_{6}-R_{5}} R(n T)+\frac{X_{6} R_{5}-X_{5} R_{6}}{R_{6}-R_{5}} \geq 0
\end{array}\right\}
$$

where $R_{n}$ and $X_{n}$ are the coordinates of the response characteristic vertices, and $R(n T)$ and $X(n T)$ are, respectively, the coordinates of the measurement resistance on the plane of complex numbers. After obtainment of the results of calculation the arrays of the values of secondary currents and voltages at the place the protection installation an array of complex values of the measurement resistances is determined by the methods of digital filtration. This array of resistances is checked if it is in the area of operation of the simulated protection. It worth mention that when the voltage at the protection installation setting place has a certain value, that is close to zero, the distance protection model increases the measurement value of voltage by a certain amount. It is necessary to ensure the correct operation of 
the distant element in case of internal and external faultsnear the place of the relay protection setting due to the need to maintain a short-term or long-term polarizing voltage, which coincides or is close in phase with the measurement voltage, which decreases to zero at close short-circuits.

In this case, the uncertainty of operation and the lack of a clear direction of protection are excluded. To date there are several options for the formation of the polarizing quantity, including using voltages of different phases. However, for correct operation in case of close three-phase short-circuits, when all voltages drop to zero, it is necessary to use a memory block with voltage fixation of the pre-emergency (load) mode.

Operation of relay protection devices depends on several factors, not only on the level of error of the current transformer: it is important to know the ratio of the magnitude of the short-circuit current near the protection installation to the operating value. It means that in one case, stable operation of the protection with a higher error is possible, and it's failure with in case of a smaller error. Therefore, the worst case of the configuration of the electrical network, as well as the value of the secondary load resistance and the type of current transformer should be considered to determine the permissible level of error. A specific algorithm was developed for this task. A certain value of the load resistance is set. At the same time, the permissible resistance of the system according to the condition of overvoltage absence in case of a fault near the place of the protection's installation is determined. In addition, the minimum possible length of electrical power transmission line, at which a ten percent error of the current transformer is observed in case of a fault at the design point, is determined. The extremum, at which the measurement resistance formed at the output of the measuring element will be as close as possible to the line of the response characteristic demarcation, is defined using the method of sequential enumeration. The intervals between the end of the measurement resistance vector and each of the faces of the response characteristics in the form of a polygon are calculated, meanwhile it is defined if the resistance vector is in range of operation. Therefore, a certain value of the parameter will correspond to a specific load resistance, by which it can be defined whether the protection device has operated or not. If the response characteristic is circle-shaped, the distance from the measuring point to the center of the response characteristic should be determined. Such calculations should be made for a range of load resistance values in order to determine the "worst" value. The corresponding algorithm is created in MATLAB software.

\section{Computational experiments}

A study of the operation of distance digital protections, namely the first stages, in CT saturation modes was carried out for this task. Two types of response characteristics are considered: a circle and a polygon. The results of the study are presented in Table 1 .
Table 1. Analisis of digital distance protection's operation.

\begin{tabular}{|c|c|c|c|c|c|c|}
\hline \multirow[t]{2}{*}{ CT type } & \multirow[t]{2}{*}{$\begin{array}{c}\mathbf{Z}_{\mathbf{n}} \\
\boldsymbol{\Omega}\end{array}$} & \multirow[t]{2}{*}{$\begin{array}{c}\mathbf{X}_{\mathrm{s}} \\
\mathbf{\Omega}\end{array}$} & \multirow[t]{2}{*}{$\begin{array}{l}\mathbf{L}_{\mathbf{L}} \\
\mathbf{k m}\end{array}$} & \multicolumn{2}{|c|}{$\begin{array}{l}\text { Intervals between the end } \\
\text { of the measurement } \\
\text { resistance vector and the } \\
\text { limit of the response } \\
\text { characteristic, } \Omega \\
\end{array}$} & \multirow[t]{2}{*}{$\begin{array}{c}\text { CT } \\
\text { error, } \\
\%\end{array}$} \\
\hline & & & & Circle & Polygon & \\
\hline $\begin{array}{c}\text { TFM- } \\
110 \\
(100 / 5) \\
\end{array}$ & 1,5 & 4,86 & 65,76 & 2.1 & 0.46 & 86 \\
\hline $\begin{array}{l}\text { TFM- } \\
110 \\
(200 / 5)\end{array}$ & 1,8 & 2,86 & 36,85 & 1.1 & 0.29 & 81 \\
\hline $\begin{array}{c}\text { TFM- } \\
110 \\
(1200 / 5)\end{array}$ & 1,8 & 0,476 & 6,14 & 0.18 & 0.047 & 83 \\
\hline
\end{tabular}

\section{Results}

If the load resistance of the CT is selected according to the conditions of infeasibility of a voltage swell in the secondary winding and $10 \%$ current error in case of fault at the computational point, then correct operation of the considered types of digital dis-tance protection is observed with all possible configurations of the electrical network. This statement refers to current transformer applications TFM-110 with different transformation ratios.

At the same time, a comparison of the operation of distant elements with different response characteristics allows to assert that it is advisable to use a relay with a circu-lar response characteristic as a high-speed line protection stage. In this case a certain margin of safety response is created (when using a polygon, the end measurement resistance vector will be located closer to the limit of the response characteristic, see Fig. 2). 

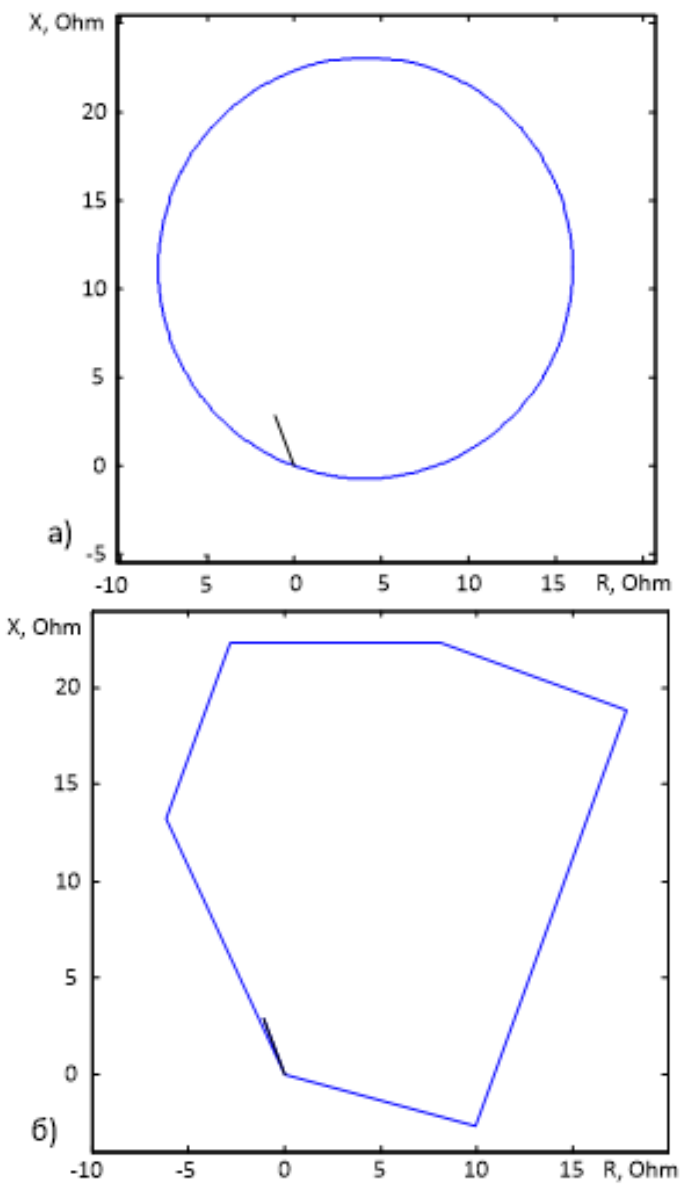

Fig. 2. Impedance protection's operation (the first line of the table 1).

This work was supported by Ministry of Education of the Novosibirsk Region (paper №2332 "The results of competitive selection for provision of targeted financial support to talented student youth - students of educational institutions of higher education located in the Novosibirsk Region, in 2020").

\section{References}

1. É. M. Shneerson, Digital Relay Protection [in Russian], Énergoatomizdat, Moscow (2007).

2. Svistunov N. A. Principles of construction systems of relay protection and automation based on microprocessor devices // izvestiya Tula State University. Technical science. - 2016. - №12-3. P. 92-95.

3. Glazyrin, V. E. Analysis of the influence of transient processes in current transformers on remote elements'behavior using mathematical models / V. E. Glazyrin, E. I. Frolova // Electro. Electrical engineering, power engineering, electrical industry. - 2012. - No. 4. - S. 9-14.

4. A Wavelet-Based Transformer Differential Protection With Differential Current Transformer Saturation and Cross-Country Fault Detection // R. Medeiros;F. Costa / IEEE Transactions on Power Delivery. - 2018. - Vol.22, Iss.2, P. 789-799. DOI: 10.1109/TPWRD.2017.2764062.
5. Kasztenny B., Mazereeuw J., Jones K. CT saturation in industrial applications - analysis and application guidelines [Electronic resource] // GE Grid Solutions. Available at: URL: https://store.gegridsolutions.com/faq/Documents/Ge neral/GET-8501.pdf. Accessed to: March 29, 2019.

6. Kuzhekov SL, Degtyarev AA. On the Coordination of Current Transformers and Relay Protection and Automation Operation of Electric-Power Systems in Transient Short-Circuit States. Electrical Engineering. 2017;12:65-72.

7. Kuzhekov S, Degtyarev A, Vorobev V. Determination of time prior saturation of the current transformers in transient short-circuit mode. Power stations. 2017;1:42-47.

8. Drozdov, AD Electric circuits with ferromagnetic elements in relay protection / AD Drozdov [and others]; ed. V.V. Platonov - 2nd ed., Revised. and add. - M.: Energoatomizdat, 1986 .-- 256 p.

9. Naumov, VA Mathematical models of current transformer in analysis of algorithms of differential protection / VA Naumov, VM Shevtsov // Elektr. stations - 2003. - No. 3. - P. 51-56.

10. Guerra, F. F. Current transformer model / F. F. Guerra, W. S. Mota // IEEE Transactions on Power Delivery. - 2007. - Vol. 22, №1 - P. 187-194.

11. U.D. Annakkage, P.G. McLaren, E. Dirks, R.P. Jayasinghe, A.D. Parker, "A current transformer model based on the Jiles-Atherton theory of ferromagnetic hysteresis", IEEE Transactions on Power Delivery, vol. 15, pp. 57-61, 2000.

12. M. Kezunovic, "Experimental Evaluation of EMTPbased Current Transformer Models for Protective Relay Transient Study", IEEE Transactions on Power Delivery, vol. 9, issue. 1, pp. 405-413, 1994.

13. I.I. Litvinov, A.A. Osintsev, V.E. Glazirin, "Characteristic features of internal and external faults for use in differential protection", Advances in Engineering Research, vol.133, pp. 425-432, 2017.

14. Glazyrin, VE Modeling of transient processes in groups of current transformers / VE Glazyrin, GE Toropov // Sb. scientific. tr. NSTU. - Novosibirsk: Publishing house of NSTU. - 2000. - No. 3 (20). - S. 75-82.

15. Sop'yanik, V. Kh. Computer calculation of transient and steady-state processes in current transformers and current circuits of the relay protection $/ \mathrm{V}$. Kh. Sop'yanik // Electric stations - 2004. - No. 2. - P. 48-52.

16. Prutik, AF Modeling of relay protection and automation in the concept of all-mode modeling of electric power systems / AF Prutik // Modern equipment and technologies: collection of articles. tr. XVI int. scientific-practical conf. students, graduate students and young scientists: in 3 volumes - Tomsk: Publishing house of Vol. polytechnic unta, 2010. - T. 1. - S. 101-102. 
17. RD 153-34.0-35.301-2002. Instructions for checking current transformers used in relay protection and measurement circuits. [in Russian] - Introduction. 03/01/2003. - M.: SPO ORGRES, 2003 .-- 152 p.

18. Korol E.G. Analysis of modeling methods of the magnetic characteristics of electromagnets for the magnetic field compensation in electrical equipment [in Russian] // Electrical Engineering and Electromechanics. - 2007. - No. 2. - p. 31-34.

19. Realization of distance protection operating characteristic / A. A. Osintsev, E. I. Frolova, A. E. Gozhenko // New in the Russian electric power industry: scientific and technical. electron. zhurn. 2017. - No. 4. - P. 64-70.

20. Recommendations for settings calculation of backup protection of high voltage transmission lines based on terminals of NPP "EKRA" [Electronic resource] // Recommendations for calculating settings. - NPP EKRA LLC, 2012. - Access mode: https://www.ekra.ru/engine/download.php?id=3738.

- Title from the screen. 